\title{
Heat shock drives genomic instability and phenotypic variations in yeast
}

\author{
Li Shen, Yu-Ting Wang, Xing-Xing Tang, Ke Zhang, Pin-Mei Wang, Yang Sui and Dao-Qiong Zheng* (i)
}

\begin{abstract}
High temperature causes ubiquitous environmental stress to microorganisms, but studies have not fully explained whether and to what extent heat shock would affect genome stability. Hence, this study explored heat-shockinduced genomic alterations in the yeast Saccharomyces cerevisiae. Using genetic screening systems and customized single nucleotide polymorphism (SNP) microarrays, we found that heat shock $\left(52^{\circ} \mathrm{C}\right)$ for several minutes could heighten mitotic recombination by at least one order of magnitude. More than half of heat-shock-induced mitotic recombinations were likely to be initiated by DNA breaks in the $S / G_{2}$ phase of the cell cycle. Chromosomal aberration, mainly trisomy, was elevated hundreds of times in heat-shock-treated cells than in untreated cells. Distinct chromosomal instability patterns were also observed between heat-treated and carbendazim-treated yeast cells. Finally, we demonstrated that heat shock stimulates fast phenotypic evolutions (such as tolerance to ethanol, vanillin, fluconazole, and tunicamycin) in the yeast population. This study not only provided novel insights into the effect of temperature fluctuations on genomic integrity but also developed a simple protocol to generate an aneuploidy mutant of yeast.
\end{abstract}

Keywords: Yeast, Heat shock, Mitotic recombination, Aneuploidy, Phenotypic evolution

\section{Key points}

Heat shock stimulates mitotic recombination and aneuploidy events in yeast.

Trisomy events outnumbers monosomy events in heat-shock-treated cells.

Heat shock and carbendazim treatment led to different patterns of genomic alterations.

Heat shock fuels phenotypic variations in yeast population.

\section{Introduction}

The yeast species Saccharomyces cerevisiae, like most cold-blooded organisms, experiences constant environmental change. Normally, yeast cells show optimal growth within a short temperature range $\left(25-30{ }^{\circ} \mathrm{C}\right)$

\footnotetext{
*Correspondence: suiyang@zju.edu.cn; zhengdaoqiong@zju.edu.cn
} Ocean College, Zhejiang University, Zhoushan 316021, Zhejiang, China
(Morano et al. 2012), and temperatures above $36{ }^{\circ} \mathrm{C}$ would trigger heat shock response in yeast cells and affect their normal physiological activities (Yamamoto et al. 2007; Morano et al. 2012; Caspeta et al. 2014). Understanding how $S$. cerevisiae responds to heat shock would not only enrich our knowledge of cell biology but would also provide references for developing robust strains for industrial application (Abdelbanat et al. 2010; Huang et al. 2018; Morard et al. 2019).

As a model organism, S. cerevisiae has been widely used to explore heat shock response modulators. Studies have reported that multiple biochemical factors contribute to yeast's tolerance and cell viability under high-temperature conditions (Morano et al. 2012). First, high temperature can greatly induce heat shock proteins (HSPs) to prevent the formation of protein aggregates and to help proteins acquire their normal functions (Piper 1995; Morano et al. 2012). Second, heat-shocktreated cells would accumulate trehalose, which stabilizes proteins and membrane (Conlin and Nelson 2007).
Springer Open

(c) The Author(s) 2020. This article is licensed under a Creative Commons Attribution 4.0 International License, which permits use, sharing, adaptation, distribution and reproduction in any medium or format, as long as you give appropriate credit to the original author(s) and the source, provide a link to the Creative Commons licence, and indicate if changes were made. The images or other third party material in this article are included in the article's Creative Commons licence, unless indicated otherwise in a credit line to the material. If material is not included in the article's Creative Commons licence and your intended use is not permitted by statutory regulation or exceeds the permitted use, you will need to obtain permission directly from the copyright holder. To view a copy of this licence, visit http://creativecommons.org/licenses/by/4.0/. 
Mutant yeast strains that are deficient in trehalose synthesis have been found to be sensitive to high temperature (Conlin and Nelson 2007; Saleh et al. 2014). Finally, antioxidant factors (superoxide dismutase, catalase, glutathione, and thioredoxin) were required to scavenge heat-shock-induced reactive oxygen species (ROS) and maintain redox homeostasis (Yamamoto et al. 2007). While research has extensively explored heat-shockinduced physiological changes (Richter et al. 2010; Morano et al. 2012), the impact of heat shock on genome integrity was less clarified. In Candida albicans, it was revealed that heat shock $\left(39{ }^{\circ} \mathrm{C}, 42{ }^{\circ} \mathrm{C}\right.$ and $50{ }^{\circ} \mathrm{C}$ ) would stimulate chromosomal aneuploidy and rearrangements (Bouchonville et al. 2009; Forche et al. 2011). However, the signature of heat-shock-induced chromosomal instability and whether similar results would be found in $S$. cerevisiae was not yet clear.

Our recent studies used the diploid S. cerevisiae strains produced by crossing two heterozygous haploid strains to determine genomic instability caused by DNA replication stress (Zheng et al. 2016; Sui et al. 2020), oxidative stress (Qi et al. 2019a, b; Zhang et al. 2019), and small molecular compounds (Qi et al. 2019a, b; Sheng et al. 2019). The abundant single nucleotide polymorphisms (SNPs) between the two homologs in the diploid strains allow for the identification of genetic events across the yeast genome at high resolution through SNP microarray and genome sequencing (Guo et al. 2017; Yin et al. 2017; Zheng and Petes 2018). In this study, heat-shock-induced genomic instability was investigated in a $S$. cerevisiae strain using genetic screening systems and customized SNP microarrays. Our findings enriched our knowledge of how temperature fluctuations affect genome integrity and phenotypic evolution.

\section{Materials and methods}

\section{Yeast strains and medium}

The $S$. cerevisiae strain JSC25-1 (MATa/MATa::HYG ade2-1/ade2-1 can1-100/can1 ura3-1/ura3 leu2-3,112/ LEU2 his3-11,15/HIS3 trp1-1/TRP1 IV1510386::KANrcan1-100/IV1510386::SUP4-o GAL2/gal2) is a diploid strain constructed by crossing of haploids isogenic to W303-1A and YJM789 (St Charles and Petes 2013). JSC25-1 is heterozygous for all SNPs that distinguish W303-1A and YJM789 except for a small region (coordinate 369,892-373,127) on chromosome (chr) XV, which is homozygous for YJM789-derived SNPs. The diploid strain JSC24-2, which has the same background as JSC251 , was used as a control in the SNP microarray analysis (St Charles and Petes 2013). The YPD medium contained $1 \%$ yeast extract, $2 \%$ peptone, and $2 \%$ glucose. To prepare solid plates, $2 \%$ agar was added into the medium, and to screen resistance mutants, YPD plates containing certain stressors $(120 \mathrm{~g} / \mathrm{L}$ ethanol, $0.1 \mathrm{~g} / \mathrm{L}$ fluconazole, $1.2 \mathrm{~g} / \mathrm{L}$ vanillin, and $4 \mathrm{mg} / \mathrm{L}$ tunicamycin) were prepared.

\section{Heat shock treatment and cell viability determination}

JSC25-1 cells were grown in a $20 \mathrm{~mL}$ YPD liquid medium with an initial $\mathrm{OD}_{600}$ of 0.05 for $16 \mathrm{~h}$. Cells were collected in PCR tubes $(50 \mu \mathrm{L})$, heated at $52{ }^{\circ} \mathrm{C}$ for $2-4 \mathrm{~min}$, and then plated on YPD plates (incubated for 3 days at $30{ }^{\circ} \mathrm{C}$ ) to determine cell viability and the rate of sectored colonies.

\section{PCR diagnosis of the left-arm heterozygosity of chr IV}

Compared with W303-1A-derived chr IV, YJM789 has a sequence deletion between 435,284 bp and 435,391 bp (Saccharomyces genome database (SGD) coordinates). Using a pair of primers (5'-AACCTTTAACATTCAGGG AG-3' and 5'-ATGACTGCTTGGTAGTTGAG-3') flanking the deletion, we can detect two DNA bands (404 bp and $296 \mathrm{bp}$ ) when the template DNA was heterozygous.

\section{Carbendazim treatment and mutant screening}

A total of 40 JSC25-1 colonies formed on the YPD plate were picked and cultured independently in $5 \mathrm{~mL}$ YPD with $25 \mathrm{mg} / \mathrm{L}$ carbendazim (Adamas-beta, Shanghai, China) for $24 \mathrm{~h}$ at $30{ }^{\circ} \mathrm{C}$ (initial $\mathrm{OD}_{600}$ of 0.05 ). The cells from each culture were washed by $\mathrm{dd}_{2} \mathrm{O}$ twice and plated on YPD plates independently to form colonies. Finally, 40 independent colonies after carbendazim treatment were selected for DNA extraction and SNP microarray analysis.

\section{Genomic DNA extraction and sonication}

Yeast cells were cultured in $7 \mathrm{~mL}$ YPD media for $24 \mathrm{~h}$ with an initial $\mathrm{OD}_{600}$ of 0.05 . For each strain, $60 \mathrm{mg}$ cells were collected through centrifugation $(5,000 \mathrm{rpm}$ for $5 \mathrm{~min}$ ) and embedded in low-melting agarose plugs with $1.2 \mathrm{mg} / \mathrm{mL}$ Zymolyase $20 \mathrm{~T}$ (Seikagaku, Tokyo, Japan). The plugs were incubated in a $1 \mathrm{~mL}$ buffer solution (500 mM EDTA, $10 \mathrm{mM}$ Tris, $\mathrm{pH} \mathrm{7.5)}$ at $37^{\circ} \mathrm{C}$ for $16 \mathrm{~h}$. Proteinase K $(2 \mathrm{mg} / \mathrm{mL})$ (Sigma-Aldrich, MO, USA) was then added to the solution, and the plugs were incubated at $50{ }^{\circ} \mathrm{C}$ for $12 \mathrm{~h}$. Finally, the plugs were washed in a $10 \mathrm{~mL}$ TE buffer ( $2 \mathrm{mM}$ Tris, $1 \mathrm{mM}$ EDTA, $\mathrm{pH}$ 8.0) at $4{ }^{\circ} \mathrm{C}$ for $48 \mathrm{~h}$ and a GeneJET PCR purification kit (Thermo Scientific, Waltham, MA, USA) was used to extract the DNA embedded in the plugs. Genomic DNA was then sonicated to fragments with an average size of 400 bp using a Bioruptor sonication device (Diagenode, Liège, Belgium).

\section{SNP microarray analysis}

To explore how heat shock affects chromosomal stability, the whole-genome SNP microarray that can 
analyze $\sim 13,000$ SNPs across the yeast genome (Charles et al. 2012) and the chr IV-specific SNP microarray (St Charles and Petes 2013) were used to detect genetic alterations in JSC25-1-derived isolates. The SNP microarrays were designed based on the SNPs between the W303-1A and YJM789 genomes (Charles et al. 2012; St Charles and Petes 2013) and were produced by Agilent (Santa Clara, CA, USA). For each selected SNP, four 25-base oligonucleotides were used: two are specific to the W303-1A-derived SNP, and the other two are specific to the YJM789-derived SNP. The SNP was situated amid the 25-base oligonucleotide. In the SNP microarray experiment, the control DNA (200 ng) extracted from the JSC24-2 cells and experimental DNA (400 ng) were labeled dUTP-Cy3 and dUTP-Cy5, respectively, using the Invitrogen BioPrime array CGH labeling system (Thermo Scientific, Waltham, MA, USA). The labeled DNAs were purified using a GeneJET PCR purification kit (Thermo Scientific, Waltham, MA, USA) and then cohybridized onto microarray slides at $62{ }^{\circ} \mathrm{C}$ for $18 \mathrm{~h}$. A GenePix 4000B scanner (Molecular Devices, Sunnyvale, CA, USA) was used to scan the slides, and GenePix 6.0 software (Molecular Devices, Sunnyvale, CA, USA) quantified the hybridization signals. The SNP microarray raw data was then entered in a GEO database (https://www.ncbi.nlm. nih.gov/geo/) with accession numbers GSE112062 and GSE150711.

\section{Stress tolerance test}

The yeast isolates were incubated in $20 \mathrm{~mL}$ YPD and a YPD medium containing a certain stressor at $30^{\circ} \mathrm{C}$ (or an indicated temperature) with an initial $\mathrm{OD}_{600}$ of 0.05 . The stress conditions were $70 \mathrm{~g} / \mathrm{L}$ ethanol, $0.15 \mathrm{mg} / \mathrm{L}$ fluconazole, $0.8 \mathrm{~g} / \mathrm{L}$ vanillin, and $1.5 \mathrm{mg} / \mathrm{L}$ tunicamycin. The biomass formation $\left(\mathrm{OD}_{600}\right)$ of yeast cells was detected using a spectrophotometer.

\section{Statistical analysis}

Fisher's exact test with a two-tailed $P$ value and a $t$-test was conducted in VassarStat (https://vassarstats.net/). Two-way hierarchical clustering analysis of these aneuploidy events was performed using the R package "pheatmap" (Kolde and Kolde 2015).

\section{Results}

\section{Heat shock stimulates genomic instability in S. cerevisiae} JSC25-1

To determine the effect of heat shock on chromosomal stability in S. cerevisiae, a sectored colony assay system was first used. S. cerevisiae JSC25-1 is homozygous for ade2-1, which displays a red phenotype because of a red pigment accumulation (a precursor of adenine) (St Charles and Petes 2013). In this strain, the ochre-suppressing tRNA mutant gene SUP4-o was inserted in the right end of the YJM789-derived chr IV (St Charles and Petes 2013). Since only one SUP4-o copy could partly suppress the ochre mutation of ade21, JSC25-1 formed pink colonies on the solid medium. Increased (two) and reduced (zero) SUP4-o copies in JSC25-1-derived isolates produce white and red colonies, respectively. Figure 1a shows a crossover event initiated by a double-strand break (DSB) on the right arm of chr IV in the first cell cycle after plating results in the white/ red-sectored JSC25-1 colonies. Break-induced replication (BIR) would produce red/pink- or white/pink-sectored colonies (Fig. 1a). This means that the frequency of sectored colonies determines the degree of chromosomal instability. Our results showed that the cell viability of JSC25-1 was reduced to $86 \%, 28 \%$, and $1.2 \%$ after heat treatment $\left(52{ }^{\circ} \mathrm{C}\right)$ duration of $3 \mathrm{~min}, 3.5 \mathrm{~min}$, and $4 \mathrm{~min}$, respectively (Fig. 1b). Without heat shock, the frequency of white/red JSC25-1 colonies was $3 \times 10^{-5}$ (Fig. 1c), and this rate was elevated 2, 4, and 11 times after heat shock for $3 \mathrm{~min}, 3.5 \mathrm{~min}$, and $4 \mathrm{~min}$, respectively (Fig. 1c). Using the PCR diagnosis described in the materials and methods section, we found that 2 of the 25 red parts of the sectored colonies came from the loss of YJM789-derived chr IV. By contrast, no chromosome loss was detected in the 25 spontaneous sectored colonies. These results suggest that heat shock can stimulate mitotic recombination and chromosome instability in yeast.

\section{Chr IV-specific SNP microarray analysis of sectored colonies}

To map the selected crossover events in the white/ red-sectored colonies, genomic DNA was extracted from the white and red sectors, respectively. The sample DNA and control DNA were competitively hybridized on a customized SNP microarray specific to chr IV (1.1 Mb, accounting for up to $10 \%$ of yeast genome) (St Charles and Petes 2013). This array includes 2,300 SNPs across the right arm of chr IV, allowing for the mapping of crossover events to about $0.5 \mathrm{~kb}$ resolution (Additional file 1: Fig. S1). In addition, other genomic regions were represented by sparse SNPs that allows for the detection of all chromosomes' aneuploidy events (Additional file 1: Fig. S1). Figure 2 shows one example of the SNP microarray results. Since a single mismatch (SNP site) is sufficient to destabilize short duplexes, the genomic DNA homozygous for the W303-1A-derived SNPs hybridizes better with the W303-1A-derived probes than with the YJM789-derived SNPs and vice versa. The blue and red lines/points in Fig. 2 indicate the hybridization level of the DNA sample from W3031A-derived and YJM789-derived SNPs, respectively. The hybridization ratio (HR) for each oligonucleotide 
a
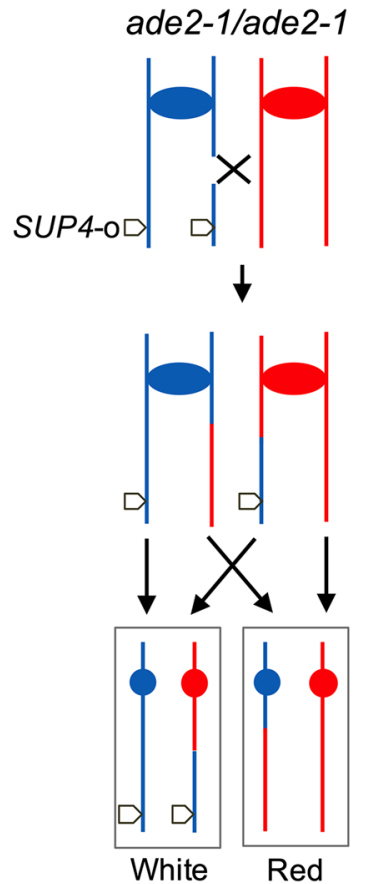

ade2-1/ade2-1
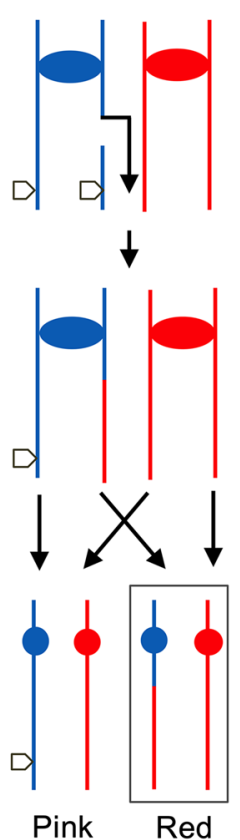

b

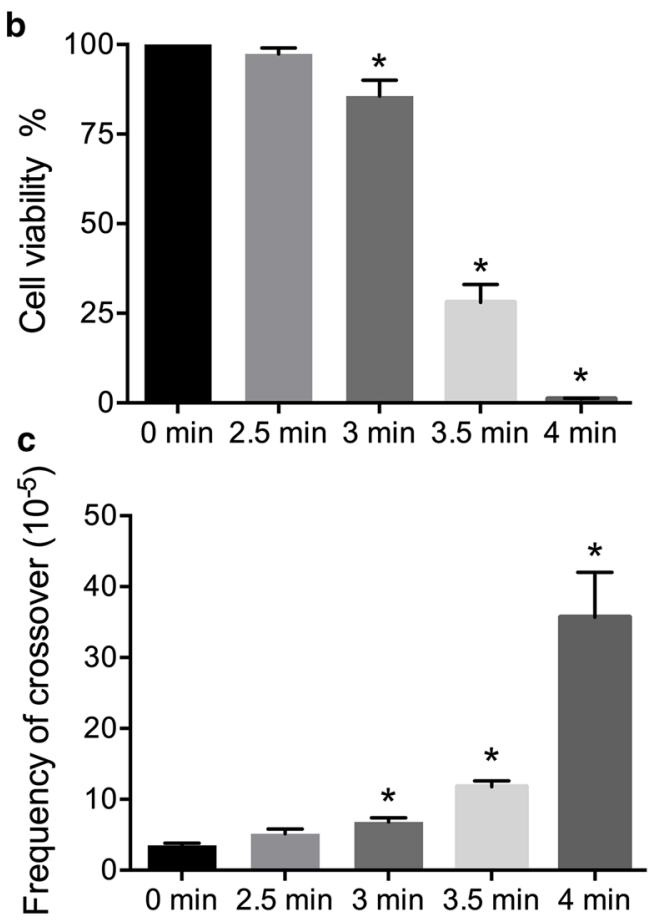

Fig. 1 Heat shock stimulates mitotic recombination in the yeast strain JSC25-1. a A reciprocal crossover event on the right arm of chromosome IV would produce white/red-sectored JSC25-1 colonies. Alternatively, break-induced replication (nonreciprocal crossover) would produce white/ pink- or red/pink-sectored colonies. b Cell viability of the JSC25-1 yeast strain after heat shock exposure (52 $\left.{ }^{\circ} \mathrm{C}\right)$ for $2-4$ min. $\mathbf{c}$ Reciprocal crossover frequency determined by calculating the red/white-sectored colonies formed on YPD plates after heat shock. "**" indicates significant difference at the level of 0.01 using $t$-test

was normalized to the $\mathrm{Cy} 5 / \mathrm{Cy} 3$ ratio of all the oligonucleotides on the microarray. HR values close to 1.5 , 1 , and 0.2 indicate 2,1 , and 0 copies of a homolog, respectively. In the white sector, the hybridization signal was transferred from heterozygosity to homozygosity (YJM789-derived homolog) between coordinates 749,244 and 750,033 (Fig. 2a, b). In the red sector, a signal transition was identified between 753,192 and 754,057 (Fig. 2c, d). For this crossover event, the region from 749,244 to 754,057 bp was identified as the crossover-associated gene conversion tract, which should include the initial recombinational lesion (Fig. 2e). Considering both sectors within the gene conversion tract, the blue SNPs were represented thrice, and the red SNPs were represented once (Fig. 2e), defined as a 3:1 pattern. As discussed in previous studies (St Charles and Petes 2013; Yin and Petes 2013; Zhang et al. 2019), such a pattern indicates that the initial recombinational lesion is likely to be a DSB occurring at one sister chromatid in the $S / G_{2}$ phase (Additional file 1: Fig. S2A). If a DSB took place in the $G_{1}$ phase, both sister chromatids would harbor DSBs at the same location after DNA replication (Additional file 1: Fig. S2B). DSB repair on both sister chromatids led to a 4:0 region within the gene conversion tract (Additional file 1: Fig. S2B). Of the 16 analyzed crossover events, 4 have no detectable conversion tracts, 7 have 3:1 pattern tracts, and 5 have complex patterns (containing 4:0 region). These results indicate that DSBs in the $S / G_{2}$ phase initiated more than half of the heat-shock-induced crossover events. It should be noted that the 4 crossover events with no detectable conversion tracts were not included in this calculation, because the phases of their initial DSBs were not identified.

Besides the crossover events on the right arm of chr IV, we also observed 8 chromosomal aneuploidy events, demonstrating the tendency of heat shock to cause chromosome aberration (Additional file 1: Table S1). We found 3 monosomic chromosomes (chr III, chr IX, and chr VI) and 5 trisomic chromosomes (chr III, chr IX, chr VI, chr VIII, and chr XVI) (Additional file 1: Table S1). As shown in Additional file 1: Table S1, we observed the monosomy of YJM789-derived chr III in the red sector and the trisomy of YJM789-derived chr III in the white sector. Such a paired event took place in three sectored colonies. These results indicate that heat shock would interrupt the normal segregation of sister chromatids and cause chromosomal nondisjunction. 

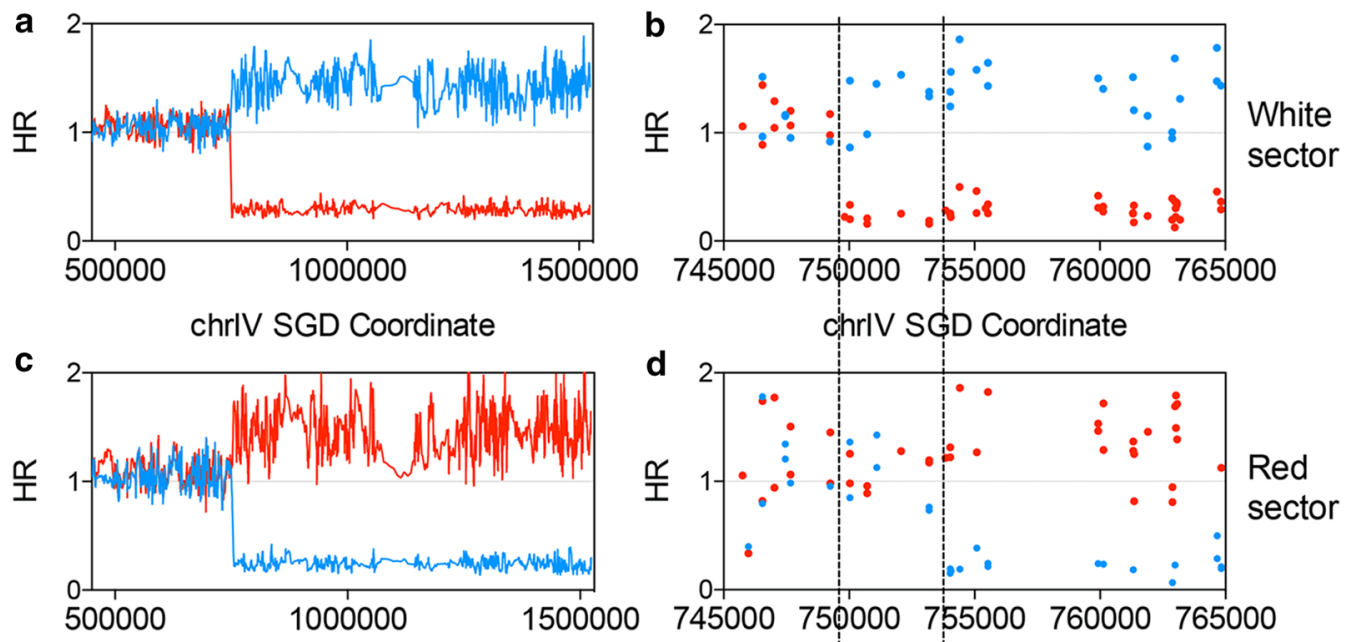

e

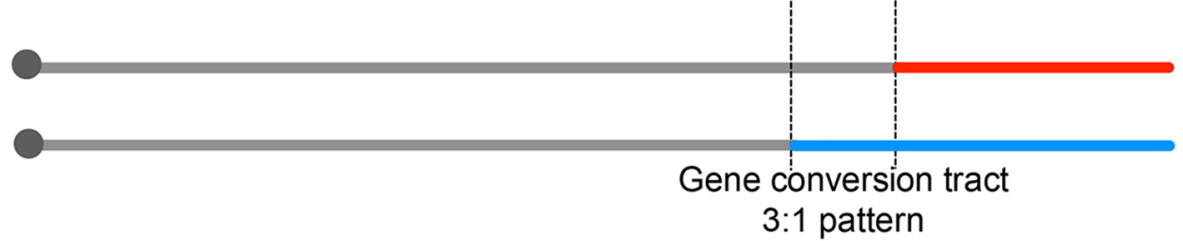

Fig. 2 Analysis of crossover events by chr IV-specific SNP microarray. The Y-axis values show the normalized hybridization ratio (HR) of genomic DNA to oligonucleotides that are specific to W303-1A- and YJM789-derived SNPs. The X-axis values indicate the SGD coordinates of the SNPs. The hybridization ratio values of about $0.2,1,1.5$ represent 0,1 , and 2 homolog copies, respectively. The red and blue lines/points indicate the HR of W303-1A- and YJM789-derived SNPS, respectively. The analysis results of the white sector and the red sector are shown in $\mathbf{a}$ and $\mathbf{b}$ at low resolution; the high-resolution results are shown in $\mathbf{c}$ and $\mathbf{d}$. e The gene conversion tract pattern associated with this crossover event

\section{Whole-genome SNP microarray analysis} of JSC25-1-derived isolates after heat shock

To explore how heat shock affects chromosomal stability at the whole-genome level, JSC25-1 cells (2 $\left.\mathrm{OD}_{600}\right)$ were heated at $52{ }^{\circ} \mathrm{C}$ for $4 \mathrm{~min}$ and plated on YPD plates to form colonies. A total of 21 colonies (named JP1-JP21) were randomly selected for SNP microarray analysis.

\section{Mitotic recombination events and loss of heterozygosity (LOH)}

In yeast, mitotic recombination is the main pathway to repair DSBs during vegetative growth, which is crucial to cell viability in the presence of DNA damage agents but inevitably leads to LOH (Symington et al. 2014). Thus, detection of $\mathrm{LOH}$ events in the heat-shock-treated yeast cells allows the determination of heat-shock-induced DSBs and mitotic recombination. As shown in Fig. 3a, we observed an increase in the signal of YJM789-derived SNPs and a decrease in the signal derived from the W303-1A homolog near $240 \mathrm{~kb}$ of chr XII. This result indicates an internal $\mathrm{LOH}$ (gene conversion) on the chr XII, which may be explained by the repair of a DSB around $240 \mathrm{~kb}$ on the W303-1A-derived chr XII using the YJM789-derived homolog as a template. In addition, we also observed that the region from $625 \mathrm{~kb}$ to the right end was homozygous for the YJM789-derived sequence. Such terminal LOH might be due to a reciprocal crossover or a BIR event, as shown in Fig. 1a. In summary, we found 17 genomic alterations, including 11 gene conversions, 5 terminal LOH events, and 1 internal deletion in the 21 JSC25-1-derived isolates after heat shock (Fig. 3b and Additional file 1: Table S2). The rate of mitotic recombination in heat-shock-treated cells was calculated at about $3 \times 10^{-2}$ events per genome per cell division (17 events/21 isolates/25 cell divisions) during the growth from a single cell to a colony on the YPD plate. Figure $3 \mathrm{~b}$ shows the patterns of all detected genetic events, and Fig. 3c presents the distribution of genetic events across 16 chromosomes. All these genetic events have unique genomic locations, indicating that none of these events took place before heat shock. Previously, O'Connell et al. (2015) detected $10 \mathrm{LOH}$ events in 10 diploid yeast isolates (isogenic to JSC25-1) that underwent 5,000 cell divisions, which means that the spontaneous rate of mitotic recombination in a wild type strain was about $2 \times 10^{-3}$ per genome per division. Our results showed that heat shock ( $52{ }^{\circ} \mathrm{C}$ for $4 \mathrm{~min}$ ) elevated the mitotic 

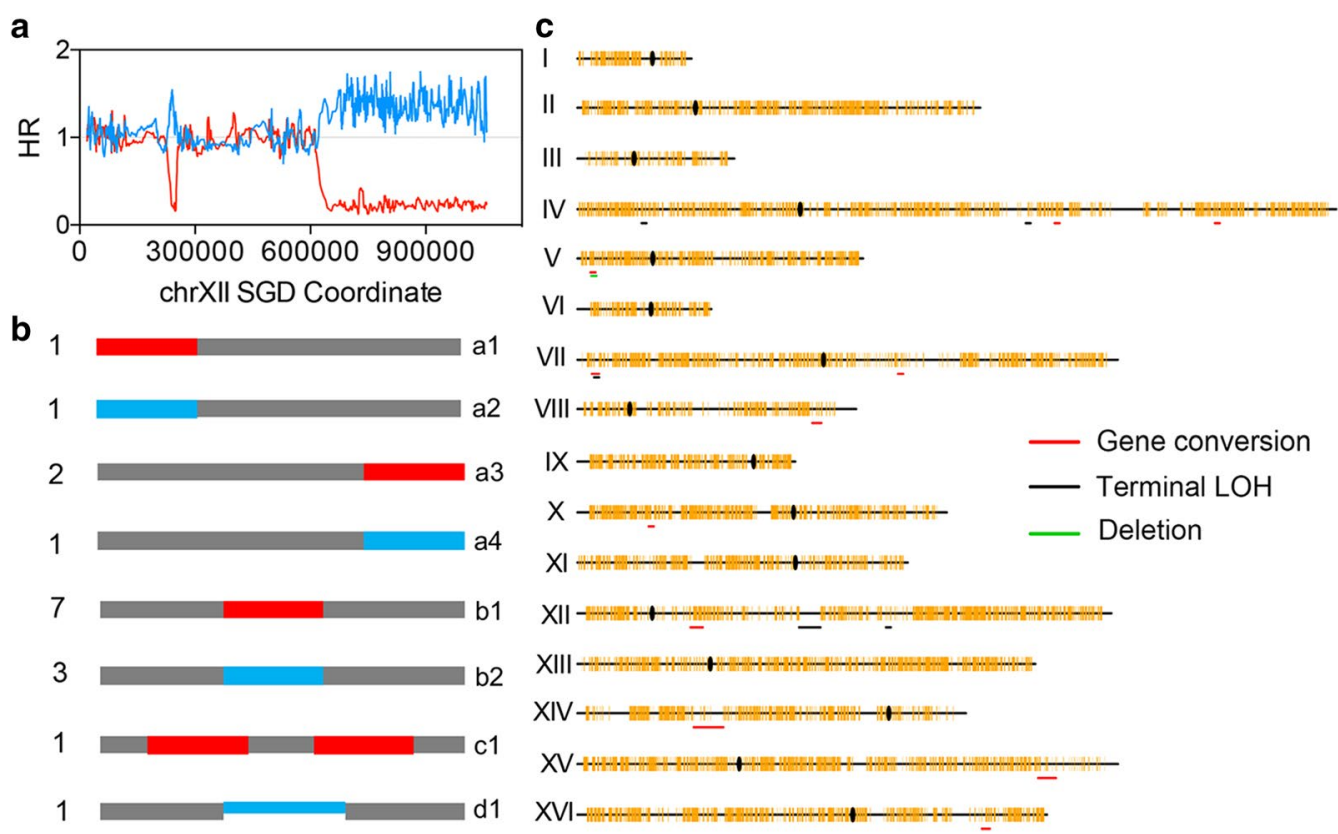

Fig. 3 Whole-genome mapping of heat-shock-induced genomic alterations in the 21 JSC25-1-derived isolates treated with heat shock. a LOH events detected on chr X in the JSC25-1-derived isolate JP14. b Patterns of genetic events. The red and blue lines represent W303-1A- and YJM789-derived homologs, respectively. The gray lines indicate heterozygous regions. Classes a1-a4 represent terminal LOH events; b1, b2 and c1 represent gene conversion events; and d1 represents deletion event. c The genetic event distribution across the 16 chromosomes. The yellow vertical lines represent SNP sites

recombination rate by at least one order of magnitude at the whole-genome level.

\section{Aneuploidy events detected in the JSC25-1-derived isolates}

Besides mitotic recombination, aneuploidy events occurred frequently in the 21 heat-shock-treated, JSC251-derived isolates. Figure $4 \mathrm{a}, \mathrm{b}$ show examples of chromosome loss (monosomy) and duplication (trisomy), respectively. In some cases, the HR values of one chromosome were reduced to 0.2 while those of the other were increased to 1.5 (Fig. 4c). This pattern indicates a uniparental disomy (UPD) event. In Fig. 4d, we showed chromosome copy number changes in the 21 isolates. There were 26 trisomic chromosomes, 4 uniparental chromosomes, 2 monosomic chromosomes, 1 pentasomic chromosome, and 1 tetrasomic chromosome. The aneuploidy event frequency was about $6.5 \times 10^{-2}$ (34 events/21 isolates/ 25 cell divisions) in the heat-shock-treated cells. At a horizontal level, the isolates with similar aneuploidy events were clustered into groups. We found that 11 of the 21 isolates had at least one aneuploidy event. In addition, trisomic chromosomes occurred at a significantly higher rate than monosomic chromosomes in the heatshock-treated yeast cells, which may be because chromosome loss is strongly and dominantly deleterious.
Zhu et al. (2014) identified 29 trisomy and 2 monosomy events in 145 wild-type diploid isolates that underwent $\sim 311,000$ cell divisions, indicating that the spontaneous rate of aneuploidy is about $1 \times 10^{-4}$ events per diploid genome per generation. Thus, our results showed an elevated chromosomal aberration rate of two orders of magnitude by heat shock.

\section{Different chromosomal instability patterns caused by heat shock and carbendazim}

We hypothesized that heat-shock-induced aneuploidy could be ascribed to the suppression of microtubule assembly dynamic. To test this hypothesis, chromosomal instability patterns caused by heat shock and carbendazim (microtubule inhibitor) were compared. Forty JSC25-1-derived isolates (MT1-MT40) were treated with $25 \mathrm{mg} / \mathrm{L}$ carbendazim for $2 \mathrm{~h}$ and then plated on YPD plates. For each isolate, only one colony was randomly selected for chr IV-specific SNP microarray analysis. In the 40 selected mutants, we observed 10 terminal $\mathrm{LOH}$ events ( 2 on chr II, 3 on chr IV, 3 on chr VII, 1 on chr X, and 1 on chr XVI). Figure 5a shows an example of these events. In the mutant MT39, we detected a deletion of the right arm of the W303-1A-derived chr III (Fig. 5b) and a duplication of the left arm of the YJM789-derived chr XIV (Fig. 5c). Such paired event is likely to reflect the 


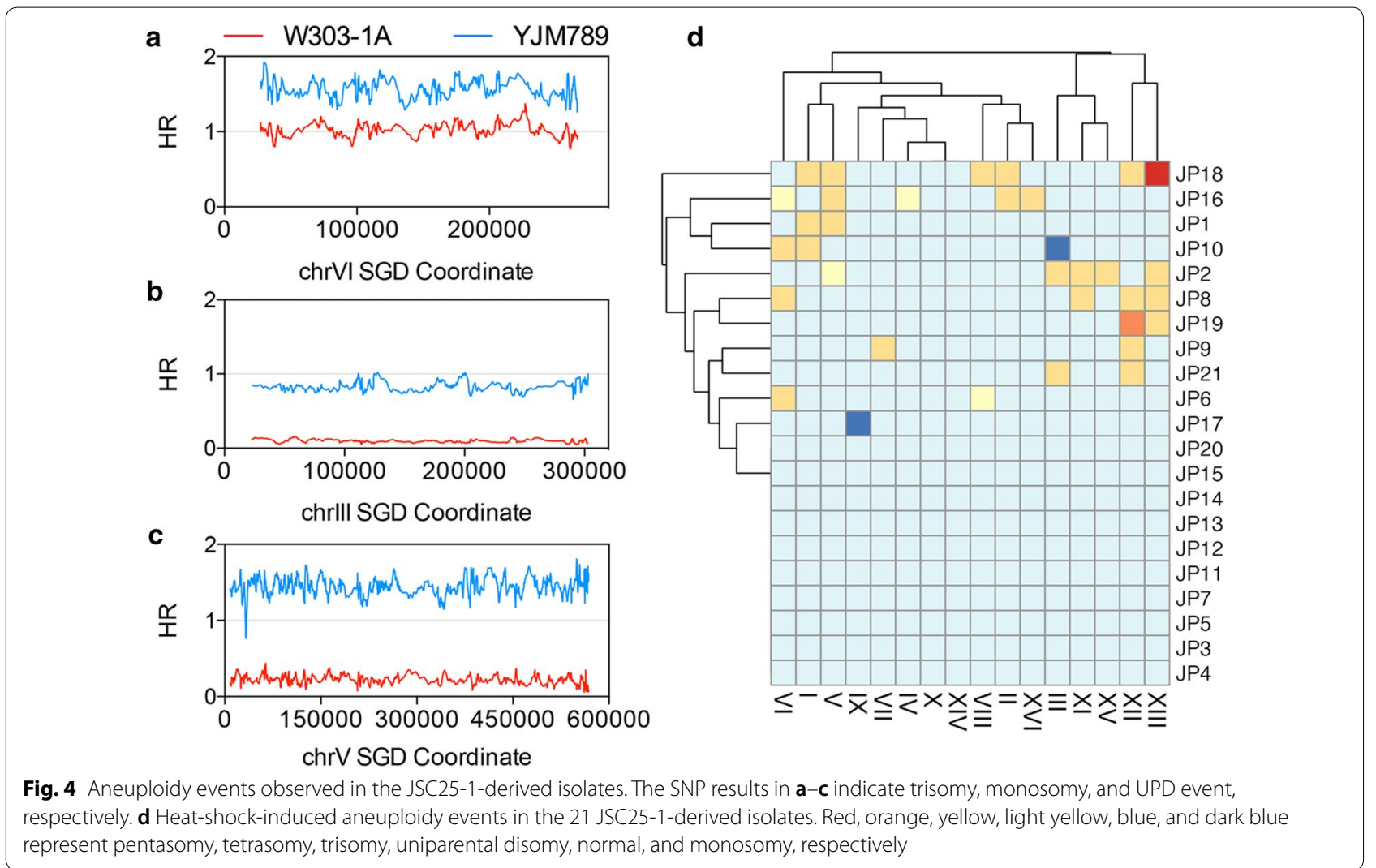

DSB repair occurring on the right arm of chr III using the left arm of chr XIV through the BIR pathway. In Fig. 5c, we showed at least one aneuploidy event in 18 mutants. In the 40 analyzed mutants, there were 28 monosomic chromosomes, 9 trisomic chromosomes, 4 tetrasomic chromosomes, and 27 UPD (Fig. 5d). Interestingly, 25 of these 27 UPD events occurred in two mutants (MT3 and MT12). The peculiar karyotypes of these two isolates suggest that carbendazim treatment readily causes UPD. Except for MT3 and MT12, other isolates tended to lose chromosomes (Fig. 5c). This result was consistent with those of previous studies showing that carbendazim exposure causes frequent chromosome loss in both yeast and mammalian cells (Wood 1982; Zuelke and Perreault 1995; Zheng et al. 2017). Using Fisher's exact test, we found that the monosomy-trisomy ratio in carbendazim-treated cells was significantly higher than that of heat-shock-treated cells. This result indicated various mechanisms underlying the carbendazim- and heatshock-induced aneuploidy events.

\section{Heat shock drives phenotypic diversification in JSC25-1}

While chromosome aneuploidy and large-scale chromosomal rearrangements are always detrimental to mammalian cells, these genetic events enhance the adaptability of yeast under certain conditions (Gilchrist and Stelkens 2019). To examine whether and to what extent heat shock can promote phenotypic changes in yeast, we compared untreated and heat-shock-treated cells in terms of the frequencies of resistance mutants that can appear on the YPD plates with stressors. Compared with untreated cells, the cells treated with heat shock showed at least 10 times more resistant colonies on the YPD plate containing $120 \mathrm{~g} / \mathrm{L}$ ethanol, $0.1 \mathrm{~g} / \mathrm{L}$ fluconazole, $1.2 \mathrm{~g} / \mathrm{L}$ vanillin, or $4 \mathrm{mg} / \mathrm{L}$ tunicamycin (Fig. 6a). Five independent isolates from different stressor-containing plates were purified on the YPD plates (from single cells to colonies). All the purified isolates from the YPD plates still showed better tolerance than that of the parental strain JSC25-1 under the test conditions (Fig. 6b), showing that heatshock-induced phenotypic variations were not caused by a transient transcriptional or post-transcriptional state. Since ethanol-resistant strains have promising applications in bioethanol production, the five mutants selected from ethanol-containing plates were analyzed using whole-genome SNP microarray. Additional file 1: Table S3 shows the genetic events on the genomes of the five mutants. Interestingly, 5 of the 7 genetic events occurred on chr IV, including 1 UPD and 4 terminal LOH (Additional file 1: Table S3). As shown in Additional file 1: 

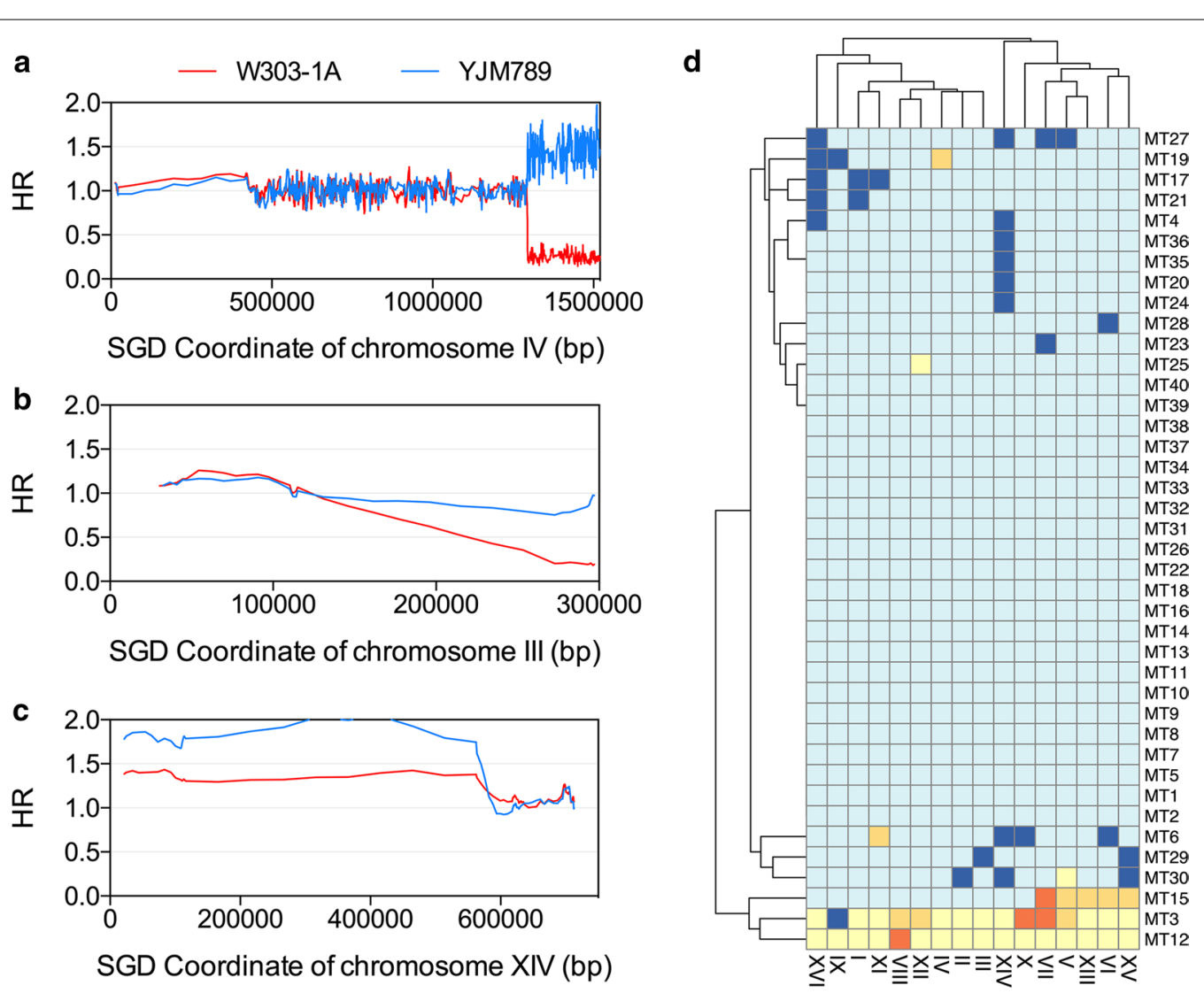

Fig. 5 Chromosomal instability resulting from carbendazim treatment in JSC25-1. Genomic alterations were detected in 40 carbendazim-treated isolates (MT1-MT40) by the chr IV-specific SNP microarray. a An example of a carbendazim-induced terminal LOH event on the right arm of chr IV. b A terminal deletion event on the right arm of chr III. c A terminal LOH duplication event on the left arm of chr XIV. The genetic events in $\mathbf{b}$ and c were observed in the same isolate. $\mathbf{d}$ Aneuploidy events resulted from carbendazim treatment. Orange, yellow, light yellow, blue, and dark blue represent tetrasomy, trisomy, uniparental disomy, normal, and monosomy, respectively

Table S3, all the five genetic events led to the homozygosity of large regions on the right arm of chr IV. A possible explanation for the improved ethanol tolerance of these five selected isolates will be discussed below.

\section{Discussion}

A sudden temperature increase creates ubiquitous environmental stress for microorganisms in both natural and industrial conditions. This study explored the effects of heat shock on genomic integrity. Our main findings are that (1) heat shock can greatly simulate chromosomal instability in yeast, (2) more than half of the heat-shockinduced $\mathrm{LOH}$ were initiated by recombinational lesions in the $S / G_{2}$ phase of the cell cycle, (3) chromosomal aberration was the most frequent genomic alteration in the heat-shock-treated yeast cells, (4) different chromosomal aberration patterns were observed between the heatshock- and carbendazim-treated cells, and (5) heat shock drives phenotypic variations in yeast populations.
In the wild-type yeast cells, mitotic recombination is the main pathway responsible for $\mathrm{LOH}$ events and largescale chromosomal rearrangements (Symington et al. 2014). We found that heat shock $\left(52{ }^{\circ} \mathrm{C}\right.$ for $4 \mathrm{~min}$ ) elevated the crossover rate by about tenfold on the right arm of chr IV in yeast (Fig. 1). In agreement with this assay, in the whole-genome SNP microarray, we found that the rate of $\mathrm{LOH}$ events in the heat-shock-treated isolates was about 15 times higher than the spontaneous rate of LOH events (O'Connell et al. 2015). By analyzing gene conversion tract patterns associated with crossover in the sectored colonies, we found that about $60 \%$ resulted from the recombinational lesion repaired in the $S / G_{2}$ phase. Previously, St. Charles and Petes showed that most $(\sim 70 \%)$ spontaneous reciprocal crossover events were initiated by DSBs in the $\mathrm{G}_{1}$ phase (St Charles and Petes 2013). In the heat-shock-treated cells, more recombinational lesions occurred in the $S / G_{2}$ phase, suggesting that the genomic DNA in the $S / G_{2}$ phase is more vulnerable to heat shock. This hypothesis was further supported 

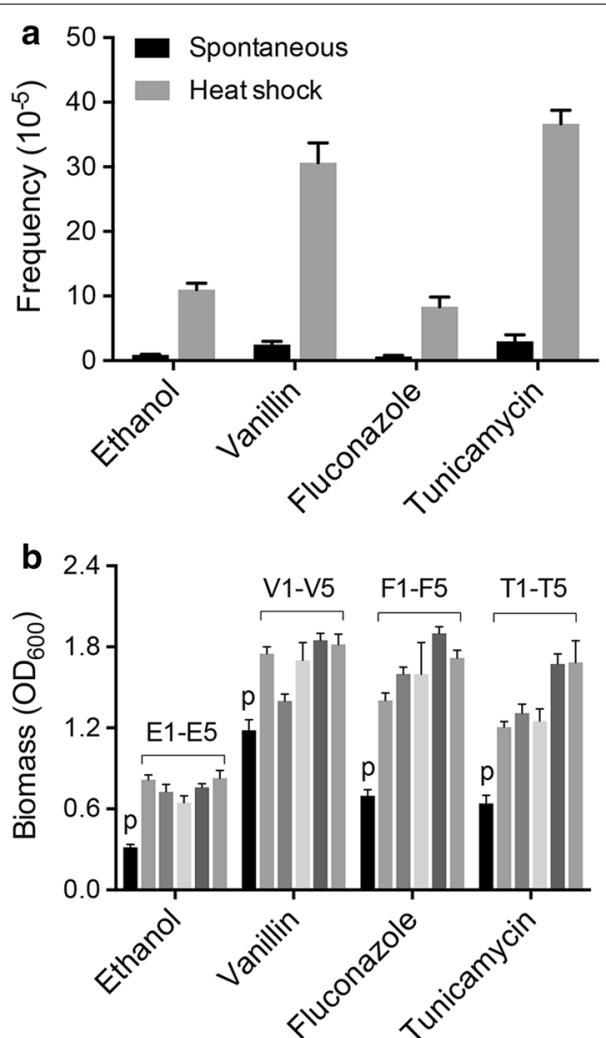

Fig. 6 Heat shock drives fast phenotypic variations in yeast population. Experiments were performed three times, and the means are shown here. Error bars represent the standard deviation. a The frequency of resistant JSC25-1 isolates appeared on the solid YPD medium containing $120 \mathrm{~g} / \mathrm{L}$ ethanol, $0.3 \mathrm{mg} / \mathrm{L}$ fluconazole, $1.2 \mathrm{~g} / \mathrm{L}$ vanillin, and $4 \mathrm{mg} / \mathrm{L}$ tunicamycin. In this experiment, about $1 \times 10^{-4}$ viable cells were plated on each plate. $\mathbf{b}$ Comparison of biomass formation $\left(\mathrm{OD}_{600}\right)$ in yeast isolates in liquid $\mathrm{YPD}$ medium containing $70 \mathrm{~g} / \mathrm{L}$ ethanol (E1-E5), $0.15 \mathrm{mg} / \mathrm{L}$ fluconazole (F1-F5), $0.8 \mathrm{~g} / \mathrm{L}$ vanillin (V1-V5), and $1.5 \mathrm{mg} / \mathrm{L}$ tunicamycin (T1-T5). The $\mathrm{OD}_{600}$ of each sample was determined at the $12 \mathrm{~h}$ time point. The JSC25-1 parental strain is indicated by " $p$ "

by the observation of heat-shock-induced DSBs in the S phase of mammalian cells (Velichko et al. 2015). Based on the established homologous recombination theory, gene conversion events mainly reflect DSB repair through homologous recombination using the synthesis-dependent strand annealing pathway while terminal $\mathrm{LOH}$ could result from the double-strand break repair pathway or BIR pathway (Symington et al. 2014; Yim et al. 2014, 2017). The rate of gene conversion and terminal $\mathrm{LOH}$ event in heat-shock-treated cells was similar with that of spontaneous $\mathrm{LOH}$ event, indicating that the choice of repairing pathway was not greatly altered by heat shock.

After entering mitosis, the replicated genome is packaged into mitotic chromosomes, with each containing two identical sister chromatids. All chromosomes are then bioriented to allow for the segregation of sister chromatids toward the mitotic poles. Spindle assembly defect and many other factors would interrupt the partitioning of the two genomes and cause chromosomal distribution errors (Quevedo et al. 2012; Germann et al. 2014). Our results showed that heat shock can elevate the aneuploidy rate by hundreds of times. The frequency of aneuploidy events $\left(6.3 \times 10^{-2}\right.$ per genome per division) was even higher than that of $\mathrm{LOH}$ events. By contrast, $\mathrm{LOH}$ events occur at a much higher rate than aneuploidy events in wild-type cells under normal conditions (O'Connell et al. 2015; Qi et al. 2019a, b). Interestingly, we found that heat shock produced more trisomic chromosomes than monosomic chromosomes in yeast, and the opposite result was found in carbendazim-treated cells (spindle assembly inhibition leads to failure in pulling the chromatid into cells). This comparison suggests that heat-shock-induced aneuploidy was not caused by spindle assembly defect. In the white/red-sectored colonies, we found that paired monosomy and trisomy occurred simultaneously in the first cell cycle after heat shock (Additional file 1: Table S1). This observation shows that heat shock would lead to a chromosomal segregation error in which both copies of the duplicated chromosome are deposited into one daughter cell and none into the other. One possible mechanism underlying heat-shockinduced chromosomal aberration is the unsuccessful cohesin breakdown, which opposes the splitting force exerted by microtubules. Although chromosomal nondisjunction results in an equal chance of trisomy and monosomy, the skewed trisomy-monosomy event ratio in heat-treated cells might be explained by the fact that trisomic chromosomes always have less of an impact on growth under normal conditions than monosomic strains (Gilchrist and Stelkens 2019). Alternatively, outnumbered trisomy events might be caused by re-replication of chromosome. It was reported that heat stress led to origin re-firing and centrosome amplification in the S phase of human HeLa cells (Petrova et al. 2016). In summary, our results demonstrated that both heat shock and carbendazim are potent inducers of chromosomal aberration. Considering different patterns can result from heat shock and carbendazim treatment, the combination of these two methods may generate more complicated karyotypes in yeast.

Aneuploidy, large-scale chromosomal rearrangements, and $\mathrm{LOH}$ are ubiquitous genomic alterations in solid tumor cells, as well as in yeast population (Zhu et al. 2016; Heil et al. 2017; Sansregret and Swanton 2017; Cho and Jinks-Robertson 2019; Gilchrist and Stelkens 2019). Many studies suggest that these genetic events would promote the adaptive evolution of yeast 
cells in response to environmental stimulus or genetic perturbations (Tan et al. 2013; Gerstein et al. 2015; Heil et al. 2017; Hose et al. 2015; Zhang et al. 2017, 2019). Based on these findings, carbendazim treatment was used in our previous study to construct robust mutants with improved tolerance to vanillin, a common inhibitor in lignocellulosic hydrolytes (Zheng et al. 2017). Our current results suggest that heat shock can develop quick adaptability to inhibitors of ethanol fermentation and antifungal drugs. Using the wholegenome SNP microarray, we found that the right arm of chr IV in several ethanol resistant isolates became homozygous to the SNPs of YJM789. In W303-1A, the gene $\operatorname{SSD} 1(1,045,640$ to $1,049,392$ on the right arm of chr IV), which encodes a translational repressor (an mRNA-binding protein that interacts with untranslated regions), is deactivated by a premature stop codon (Avrahami-Moyal et al. 2012). This mutation reduces competitive fitness and increases sensitivity to ethanol and high temperature (Avrahami-Moyal et al. 2012; Hose et al. 2020). Thus, it is highly probable that the genetic events (including both aneuploidy and crossover events) resulting in the homozygosity of YJM789derived SSD1 were positively selected after heat shock and contributed to the ethanol tolerance of yeast as well.

To the best of our knowledge, this study is the first to show data demonstrating that heat shock could significantly stimulate chromosomal instability at the wholegenome level and phenotypic diversification in yeast. We also provided a convenient and efficient method to generate aneuploidy yeast mutants with potential in basic research as well as in industrial application.

\section{Supplementary information}

Supplementary information accompanies this paper at https://doi. org/10.1186/s13568-020-01091-7.

Additional file 1. Additional figures and tables.

\section{Acknowledgements}

This work was supported by National Natural Science Foundation of China (31800055), Natural Science Foundation of Zhejiang Province (LY18C060002), and Zhoushan City-Zhejiang University Joint Specific Project (2019C81055). We thank Thomas D. Petes (Duke University) for technological supports of SNP microarray analysis.

\section{Authors' contributions}

LS, YS, and D-QZ conceived and designed research. LS, Y-TW, X-XT, YS, and KZ conducted experiments. LS, YS, D-QZ, and P-MW analyzed data. LS, YS, and

D-QZ wrote the manuscript. All authors read and approved the manuscript.

\section{Funding}

This study was funded by National Natural Science Foundation of China (31800055), Natural Science Foundation of Zhejiang Province (LY18C060002), and Zhoushan City-Zhejiang University Joint Specific Project (2019C81055).

\section{Availability of data and materials}

The SNP microarray raw data was then entered in a GEO database (https ://www.ncbi.nlm.nih.gov/geo/) with accession numbers GSE112062 and GSE150711.

\section{Ethics approval and consent to participate}

This article does not contain any studies with human participants or animals performed by any of the authors.

\section{Consent for publication}

All authors agreed to publish our paper in AMB Express.

\section{Competing interests}

The authors declare that they have no competing interests.

Received: 6 August 2020 Accepted: 11 August 2020

Published online: 17 August 2020

\section{References}

Abdelbanat BMA, Hoshida H, Ano A, Nonklang S, Akada R (2010) Hightemperature fermentation: how can processes for ethanol production at high temperatures become superior to the traditional process using mesophilic yeast? Appl Microbiol Biotechnol 85:861-867. https://doi. org/10.1007/s00253-009-2248-5

Avrahami-Moyal L, Braun S, Engelberg D (2012) Overexpression of PDE2 or SSD1-V in Saccharomyces cerevisiae W303-1A strain renders it ethanol-tolerant. FEMS Yeast Res 12:447-455. https://doi.org/10.111 1/j.1567-1364.2012.00795.x

Bouchonville K, Forche A, Tang KE, Selmecki A, Berman J (2009) Aneuploid chromosomes are highly unstable during DNA transformation of Candida albicans. Eukaryot Cell 8:1554-1566. https://doi.org/10.1128/EC.00209-09

Caspeta L, Chen Y, Ghiaci P, Feizi A, Buskov S, Hallstrom BM, Petranovic D, Nielsen J (2014) Altered sterol composition renders yeast thermotolerant. Science 346:75-78. https://doi.org/10.1126/science.1258137

Charles JS, Hazkani-Covo E, Yin Y, Andersen SL, Dietrich FS, Greenwell PW, Malc E, Mieczkowski P, Petes TD (2012) High-resolution genome-wide analysis of irradiated (UV and gamma rays) diploid yeast cells reveals a high frequency of genomic loss of heterozygosity $(\mathrm{LOH})$ events. Genetics 190:1267-1284. https://doi.org/10.1534/genetics.111.137927

Cho J-E, Jinks-Robertson S (2019) Deletions associated with stabilization of the Top1 cleavage complex in yeast are products of the nonhomologous end-joining pathway. Proc Natl Acad Sci USA 116:22683-22691. https:// doi.org/10.1073/pnas.1914081116

Conlin LK, Nelson HCM (2007) The natural osmolyte trehalose is a positive regulator of the heat-induced activity of yeast heat shock transcription factor. Mol Cell Biol 27:1505-1515. https://doi.org/10.1128/MCB.01158-06

Forche A, Abbey D, Pisithkul T, Weinzierl MA, Ringstrom T, Bruck D, Petersen K, Berman J (2011) Stress alters rates and types of loss of heterozygosity in Candida albicans. mBio 2:e00129-00111. https://doi.org/10.1128/ mBio.00129-11

Germann SM, Schramke V, Pedersen RT, Gallina I, Eckert-Boulet N, Oestergaard VH, Lisby M (2014) TopBP1/Dpb11 binds DNA anaphase bridges to prevent genome instability. J Cell Biol 204:45-59. https://doi.org/10.1083/ jcb.201305157

Gerstein AC, Fu MS, Mukaremera L, Li Z, Ormerod KL, Fraser JA, Berman J, Nielsen K (2015) Polyploid titan cells produce haploid and aneuploid progeny to promote stress adaptation. mBio 6:e01340-15. https://doi. org/10.1128/mBio.01340-15

Gilchrist C, Stelkens R (2019) Aneuploidy in yeast: segregation error or adaptation mechanism? Yeast 36:525-539. https://doi.org/10.1002/yea.3427

Guo X, Hum YF, Lehner K, Jinks-Robertson S (2017) Regulation of hetDNA length during mitotic double-strand break repair in yeast. Mol Cell 67:539-549. https://doi.org/10.1016/j.molcel.2017.07.009

Heil CSS, DeSevo CG, Pai DA, Tucker CM, Hoang ML, Dunham MJ (2017) Loss of heterozygosity drives adaptation in hybrid yeast. Mol Biol Evol 34:1596-1612. https://doi.org/10.1093/molbev/msx098 
Hose J, Yong CM, Sardi M, Wang Z, Newton MA, Gasch AP (2015) Dosage compensation can buffer copy-number variation in wild yeast. eLife 4:e05462. https://doi.org/10.7554/eLife.52063

Hose J, Escalante LE, Clowers KJ, Dutcher HA, Robinson D, Bouriakov V, Coon JJ, Shishkova E, Gasch AP (2020) The genetic basis of aneuploidy tolerance in wild yeast. eLife 9:e52063. https://doi.org/10.7554/eLife.52063

Huang CJ, Lu M, Chang Y, Li W (2018) Experimental evolution of yeast for high-temperature tolerance. Mol Biol Evol 35:1823-1839. https://doi. org/10.1093/molbev/msy077

Kolde R, Kolde MR (2015) Package 'pheatmap'. R Package 1:790

Morano KA, Grant CM, Moye-Rowley WS (2012) The response to heat shock and oxidative stress in Saccharomyces cerevisiae. Genetics 190:1157-1195. https://doi.org/10.1534/genetics.111.128033

Morard M, Macias LG, Adam AC, Lairon-Peris M, Perez-Torrado R, Toft C, Barrio E (2019) Aneuploidy and ethanol tolerance in Saccharomyces cerevisiae. Front Genet 10:82. https://doi.org/10.3389/fgene.2019.00082

O'Connell K, Jinks-Robertson S, Petes TD (2015) Elevated genome-wide instability in yeast mutants lacking RNase H activity. Genetics 201:963-975. https://doi.org/10.1534/genetics.115.182725

Petrova NV, Velichko AK, Razin SV, Kantidze OL (2016) Early S-phase cell hypersensitivity to heat stress. Cell Cycle 15:337-344. https://doi. org/10.1080/15384101.2015.1127477

Piper PW (1995) The heat shock and ethanol stress responses of yeast exhibit extensive similarity and functional overlap. FEMS Microbiol Lett 134:121127. https://doi.org/10.1111/j.1574-6968.1995.tb07925.x

Qi L, Wu XC, Zheng DQ (2019a) Hydrogen peroxide, a potent inducer of global genomic instability. Curr Genet 65:913-917. https://doi.org/10.1007/ s00294-019-00969-9

Qi L, Zhang K, Wang YT, Wu JK, Sui Y, Liang XZ, Yu LZ, Wu XC, Wang PM, Xu JZ, Zheng DQ (2019b) Global analysis of furfural-induced genomic instability using a yeast model. Appl Environ Microbiol 85:e01237-e1319. https:// doi.org/10.1128/AEM.01237-19

Quevedo O, Garcia-Luis J, Matos-Perdomo E, Aragon L, Machin F (2012) Nondisjunction of a single chromosome leads to breakage and activation of DNA damage checkpoint in G2. PLoS Genet 8:e1002509. https://doi. org/10.1371/journal.pgen.1002509

Richter K, Haslbeck M, Buchner J (2010) The heat shock response: life on the verge of death. Mol Cell 40:253-266. https://doi.org/10.1016/j.molce I.2010.10.006

Saleh AA, Gune US, Chaudhary RK, Turakhiya AP, Roy I (2014) Roles of Hsp104 and trehalose in solubilisation of mutant huntingtin in heat shocked Saccharomyces cerevisiae cells. Biochim Biophys Acta 1843:746-757. https:// doi.org/10.1016/j.bbamcr.2014.01.004

Sansregret L, Swanton C (2017) The role of aneuploidy in cancer evolution. Cold Spring Harb Perspect Med 7:a028373. https://doi.org/10.1101/cshpe rspect.a028373

Sheng H, Qi L, Sui Y, Li YZ, Yu LZ, Zhang K, Xu JZ, Wang PM, Zheng DQ (2019) Mapping chromosomal instability induced by small-molecular therapeutics in a yeast model. Appl Microbiol Biotechnol 103:4869-4880. https:// doi.org/10.1007/s00253-019-09845-5

St Charles J, Petes TD (2013) High-resolution mapping of spontaneous mitotic recombination hotspots on the $1.1 \mathrm{Mb}$ arm of yeast chromosome IV. PLoS Genet 9:e1003434. https://doi.org/10.1371/journal.pgen.1003434

Sui Y, Qi L, Zhang K, Saini N, Klimczak LJ, Sakofsky CJ, Gordenin DA, Petes TD, Zheng D-Q (2020) Analysis of APOBEC-induced mutations in yeast strains with low levels of replicative DNA polymerases. Proc Natl Acad Sci USA 117:9440-9450. https://doi.org/10.1073/pnas.1922472117

Symington LS, Rothstein R, Lisby M (2014) Mechanisms and regulation of mitotic recombination in Saccharomyces cerevisiae. Genetics 198:795835. https://doi.org/10.1534/genetics.114.166140
Tan Z, Hays M, Cromie GA, Jeffery EW, Scott AC, Ahyong V, Sirr A, Skupin A, Dudley AM (2013) Aneuploidy underlies a multicellular phenotypic switch. Proc Natl Acad Sci USA 110:12367-12372. https://doi. org/10.1073/pnas.1301047110

Velichko AK, Petrova NV, Razin SV, Kantidze OL (2015) Mechanism of heat stress-induced cellular senescence elucidates the exclusive vulnerability of early S-phase cells to mild genotoxic stress. Nucleic Acids Res 43:6309-6320. https://doi.org/10.1093/nar/gkv573

Wood JS (1982) Genetic effects of methyl benzimidazole-2-yl-carbamate on Saccharomyces cerevisiae. Mol Cell Biol 2:1064-1079. https://doi. org/10.1128/MCB.2.9.1064

Yamamoto A, Ueda J, Yamamoto N, Hashikawa N, Sakurai H (2007) Role of heat shock transcription factor in Saccharomyces cerevisiae oxidative stress response. Eukaryot Cell 6:1373-1379. https://doi.org/10.1128/EC.00098 $-07$

Yim E, O'Connell KE, Charles JS, Petes TD (2014) High-resolution mapping of two types of spontaneous mitotic gene conversion events in Saccharomyces cerevisiae. Genetics 198:181-192. https://doi.org/10.1534/genet ics.114.167395

Yin Y, Petes TD (2013) Genome-wide high-resolution mapping of UV-induced mitotic recombination events in Saccharomyces cerevisiae. PLoS Genet 9:e1003894. https://doi.org/10.1371/journal.pgen.1003894

Yin Y, Dominska M, Yim E, Petes TD (2017) High-resolution mapping of heteroduplex DNA formed during UV-induced and spontaneous mitotic recombination events in yeast. eLife 6:e28069. https://doi.org/10.7554/ elife.28069

Zhang K, Fang Y-H, Gao K-H, Sui Y, Zheng D-Q, Wu X-C (2017) Effects of genome duplication on phenotypes and industrial applications of Saccharomyces cerevisiae strains. Appl Microbiol Biotechnol 101:5405-5414. https://doi.org/10.1128/mBio.02099-17

Zhang K, Zheng DQ, Sui Y, Qi L, Petes TD (2019) Genome-wide analysis of genomic alterations induced by oxidative DNA damage in yeast. Nucleic Acids Res 47:3521-3535. https://doi.org/10.1093/nar/gkz027

Zheng D-Q, Petes TD (2018) Genome instability induced by low levels of replicative DNA polymerases in yeast. Genes 9:539. https://doi.org/10.3390/ genes9110539

Zheng DQ, Zhang K, Wu XC, Mieczkowski PA, Petes TD (2016) Global analysis of genomic instability caused by DNA replication stress in Saccharomyces cerevisiae. Proc Natl Acad Sci USA 113:E8114-E8121. https://doi. org/10.1073/pnas.1618129113

Zheng DQ, Jin XN, Zhang K, Fang YH, Wu XC (2017) Novel strategy to improve vanillin tolerance and ethanol fermentation performances of Saccharomycere cerevisiae strains. Bioresour Technol 231:53-58. https://doi. org/10.1016/j.biortech.2017.01.040

Zhu YO, Siegal ML, Hall DW, Petrov DA (2014) Precise estimates of mutation rate and spectrum in yeast. Proc Natl Acad Sci USA 111:E2310-E2318. https://doi.org/10.1073/pnas.1323011111

Zhu YO, Sherlock G, Petrov DA (2016) Whole genome analysis of 132 clinical Saccharomyces cerevisiae strains reveals extensive ploidy variation. G3 6:2421-2434. https://doi.org/10.1534/g3.116.029397

Zuelke KA, Perreault SD (1995) Carbendazim (MBC) disrupts oocyte spindle function and induces aneuploidy in hamsters exposed during fertilization (meiosis II). Mol Reprod Dev 42:200-209. https://doi.org/10.1002/ mrd.1080420209

\section{Publisher's Note}

Springer Nature remains neutral with regard to jurisdictional claims in published maps and institutional affiliations. 